\title{
Effect of neodymium doping on structural, microscopic and electrical properties of barium phosphate system
}

\author{
Shivani Suri, Vishal Singh and K. K. Bamzai* \\ Crystal Growth and Materials Research Laboratory \\ Department of Physics and Electronics \\ University of Jammu, Jammu 180006, India \\ *kkbamz@yahoo.com
}

Received 30 June 2013; Revised 20 March 2014; Accepted 24 March 2014; Published 7 May 2014

\begin{abstract}
Neodymium-doped barium phosphate (NdBP) was prepared as single crystal by room temperature solution technique known as gel encapsulation technique. Single crystal X-ray diffraction shows that the crystal belongs to orthorhombic system. The flower type morphology was observed by scanning electron microscope (SEM) and the stoichiometric composition of the prepared crystal was observed by energy dispersive X-ray analysis (EDAX). The presence of functional group and other groups was studied by Fourier transform infrared spectroscopy (FTIR). The electrical properties of these materials like dielectric constant $\left(\varepsilon^{\prime}\right)$, dielectric loss $(\tan \delta)$ and ac conductivity $\left[\ln \left(\sigma_{\mathrm{ac}}\right)\right]$ was studied at different temperatures ranging from $40^{\circ} \mathrm{C}$ to $420^{\circ} \mathrm{C}$ in the frequency range of $5 \mathrm{kHz}$ to $1 \mathrm{MHz}$. The activation energy values decreases with increase in frequency suggesting that the conduction mechanism is because of hopping of charge carriers.
\end{abstract}

Keywords: Inorganic compounds; crystal growth; X-ray diffraction; dielectric properties.

\section{Introduction}

The alkaline earth phosphates has received enormous importance for their use as phosphor matrices ${ }^{1}$ and are regarded as technically very potential and promising material. Among the family of phosphate, potassium dihydrogen phosphate (KDP) is one of the best known dielectric, ferroelectric material which exhibit excellent quadratic nonlinearity. KDP crystal is well known for their good piezoelectric and nonlinear optical properties, that draw much attention for their significant theoretical interests and commercial applications in the optical modulation, frequency conversions and electrooptical devices. ${ }^{2,3}$ Therefore, it is worthwhile to study the electrical properties of neodymium-doped barium phosphate (NdBP) which also belongs to phosphate family. Phosphates being sparingly soluble in water have been grown by gel method. ${ }^{4}$ The present paper reports the growth and characterization of rare earth doping on BP system. For the selection of dopant, ionic radii have been considered as an important parameter. The dopant ion $\mathrm{Nd}$ has been selected for the modification because the ionic radii of $\mathrm{Nd}^{3+}$ ion $(1.08 \AA)$ is close to that of $\mathrm{Ba}^{2+}$ ion $(1.35 \AA)$. Ions of the same size and same charge easily replace each other but effect the unit cell size of the parent component. According to Goldsmith rule, the dopant cations enter into the sites if radius of the substituted ion and replaced ion does not differ by $15-20 \% .^{5}$ So, the effect of substitution on various characteristics of parent material can be unfolded by carrying out systematic study of the modified composition. Authors have already reported the growth, thermal kinetics and dielectric as well as ac conductivity studies of pure and cadmium-doped barium phosphate single crystal. ${ }^{6,7}$ In the present paper, we are reporting synthesis, characterization and detailed electrical properties of NdBP system and correlating the results with our earlier reports of pure BP crystals. To the best of author's knowledge, there is no such report on the effect of $\mathrm{Nd}$ doping on the BP system.

\section{Experimental Technique}

\subsection{Synthesis of NdBP}

NdBP in the form of single crystal was synthesized by controlled diffusion of chemical reagents through the silica gel. The system used for the synthesis was $\mathrm{BaCl}_{2}-\mathrm{Nd}\left(\mathrm{NO}_{3}\right)_{3}-$ $\mathrm{H}_{3} \mathrm{PO}_{4}-\mathrm{Na}_{2} \mathrm{SiO}_{3}$. The high quality pure grade chemicals from S D Fine Chemical Ltd used are barium chloride (99\% AR), orthophosphoric acid ( $85 \%$ LR), neodymium nitrate (99\% AR) and sodium metasilicate (99\% AR). The crystallization apparatus used is a test tube of length $20 \mathrm{~cm}$ and diameter $2.5 \mathrm{~cm}$. Silica gel used for the growth experiment is optically transparent and is obtained by the neutralization of sodium metasilicate. The gelling reaction is based on the hydrolytic process given by the following chemical reaction:

$$
\mathrm{Na}_{2} \mathrm{SiO}_{3}+\mathrm{H}_{2} \mathrm{O} \rightarrow \mathrm{H}_{4} \mathrm{SiO}_{4}+2 \mathrm{NaOH} \text {. }
$$

Silica gel was prepared by dissolving $212.14 \mathrm{~g}$ of sodium metasilicate $\left(\mathrm{Na}_{2} \mathrm{SiO}_{3}\right)$ in $1000 \mathrm{ml}$ of distilled water to have a

This is an Open Access article published by World Scientific Publishing Company. It is distributed under the terms of the Creative Commons Attribution 3.0 (CC-BY) License. Further distribution of this work is permitted, provided the original work is properly cited. 
gel of 1 molar concentration. The sodium metasilicate solution was then impregnated with orthophosphoric acid in accordance with $\mathrm{pH}$ value with continuous stirring in order to avoid any local ion concentration which would otherwise cause premature local gelling and make final solution inhomogeneous. The $\mathrm{pH}$ of the resulting mixture was measured using digital $\mathrm{pH}$ meter immediately after proper mixing. The $\mathrm{pH}$ value of the solution was adjusted in the value ranging from 4 to 7. A gel solution of fixed amount with desired $\mathrm{pH}$ value was then allowed to set in the crystallizer. The gel setting time was found to be strongly $\mathrm{pH}$ dependent and environmental temperature. It may take about $24 \mathrm{~h}$ for the gel to set in summer $\left(35-40^{\circ} \mathrm{C}\right)$ whereas in winter $\left(10-15^{\circ} \mathrm{C}\right)$ it would take even 14 days for the gel to set. The growth of NdBP was accomplished by the controlled diffusion of $\mathrm{Nd}^{3+}$ and $\mathrm{Ba}^{2+}$ ions through silica gel impregnated with orthophosphoric acid. A mixture of $0.9 \mathrm{M}$ barium chloride $\left(\mathrm{BaCl}_{2}\right)$ and $0.1 \mathrm{M}$ neodymium nitrate $\left[\mathrm{Nd}\left(\mathrm{NO}_{3}\right)_{3}\right]$ in the ratio of $1: 1$ by volume was gently poured over the perfectly set gel along with walls of the test tube so as to avoid gel breakage. The proposed chemical reaction for the growth of $\mathrm{NdBP}$ crystals is:

$$
\begin{aligned}
& 3 \mathrm{BaCl}_{2} \cdot 2 \mathrm{H}_{2} \mathrm{O}+3 \mathrm{Nd}\left(\mathrm{NO}_{3}\right)_{3} \cdot 6 \mathrm{H}_{2} \mathrm{O}+2 \mathrm{H}_{3} \mathrm{PO}_{4} \\
& \rightarrow \mathrm{Nd}_{3}: \mathrm{Ba}_{3}\left(\mathrm{PO}_{4}\right)_{2} \cdot \mathrm{H}_{2} \mathrm{O}+6 \mathrm{HCl}+9 / 2 \mathrm{O}_{2}+9 \mathrm{NO}_{2}
\end{aligned}
$$

\subsection{Characterization}

Morphological studies were carried out using scanning electron microscope (SEM) model JEOL 840, whereas structural investigation was done by using both single crystal (Oxford X-ray diffractometer) and powder X-ray diffraction (XRD) (Rigaku Co. Ltd. Japan) with $\mathrm{Cu} \mathrm{K}_{\alpha}$ radiation $(\lambda=1.54 \AA$ ) with a scanning rate of $2 \% \mathrm{~min}$. Energy dispersive $\mathrm{X}$-rays analysis (EDAX) was recorded using dispersive spectrometer (INCA ENERGY EDAX) attached to the SEM for carrying out elemental analysis. Fourier transform infra-red (FTIR) spectra was recorded in the wave number range of $4000-400 \mathrm{~cm}^{-1}$ by the Perkin-Elmer 781 spectrophotometer using $\mathrm{KBr}$ pellet method. The dielectric studies were carried out with the help of automated impendence analyzer (LF 4192A model) interfaced with USB GPIB converter $82357 \mathrm{~B}$ (Agilent) and further automated by using a computer for data recording, storage and analysis. For dielectric studies, since the size of the grown crystal was small, so the crystals were first powdered in an agate mortar and then about 1-2\% PVA (poly vinyl alcohol) was added which served as a binder. The fine powder was then pressed into circular pellets of $13 \mathrm{~mm}$ diameter and thickness of $0.65 \mathrm{~mm}$ using a hydraulic press by applying a pressure of 10 ton. The circular pellets were then heated up to a temperature of $250^{\circ} \mathrm{C}$ slowly at a heating rate of $2{ }^{\circ} \mathrm{C} / \mathrm{min}$ before performing any measurement in order to overcome the effect of binder. The conducting silver paste was applied on both sides of the circular pellet which acted as capacitor with the material as dielectric. The pre-heated sample was then mounted in specially designed two terminal sample holder. The instrument directly provides the value of capacitance $(C)$ and dielectric loss $(\tan \delta)$. The dielectric constant was calculated using the relation:

$$
\varepsilon^{\prime}=C t / \varepsilon_{0} A
$$

where ' $C$ ' is the capacitance in $\mathrm{F}$, ' $t$ ' is the thickness (m), ' $A$ ' is the area of cross section $\left(\mathrm{m}^{2}\right)$ of sample and ' $\varepsilon_{0}$ ' is the absolute permittivity of the free space having value of $8.854 \times 10^{-12} \mathrm{Fm}^{-1}$.

The ac conductivity $\left(\sigma_{\text {ac }}\right)$ was calculated using the relation $\sigma_{\mathrm{ac}}=2 \pi f \varepsilon_{0} \varepsilon \tan \delta$, where ' $f$ ' is the frequency of the applied ac field $(\mathrm{Hz})$. The activation energy was calculated from the Arrhenius graph of $\left(\ln \sigma_{\mathrm{ac}}\right)$ versus inverse of temperature using the relation ${ }^{8} \sigma=\sigma_{0} \exp \left[-\frac{E_{a}}{R T}\right]$, where ' $\sigma$ ' is a conductance at temperature ' $T$ ' and ' $R$ ' the gas constant $(R=$ $8.314472 \mathrm{~mol}^{-1} \mathrm{~K}^{-1}$ ).

\section{Results and Discussion}

\subsection{Synthesis}

The upper reactant i.e., a mixture of $\mathrm{BaCl}_{2}$ and $\mathrm{Nd}\left(\mathrm{NO}_{3}\right)_{3}$ of 1 molar concentration in the ratio of $1: 1$ by volume when poured over a perfectly set gel, then the anions of upper reactant i.e., $\mathrm{Nd}^{3+}$ and $\mathrm{Ba}^{2+}$ slowly diffuses into the gel column containing phosphate ions. As a result, the light pink precipitates were formed instantaneously there by suggesting spontaneous nucleation. The precipitates are the solid phase formed within the liquid phase. Therefore, crystallization is an aspect of precipitation obtained through the variation of solubility conditions of the solute in the solvent. After $24 \mathrm{~h}$, small shining crystals appear below the precipitate band and as the time increases, the thickness of the precipitation band increases gradually as the diffusion proceeds into the gel. After a few days, the distance between the precipitate band and crystal increases with the increase in number and size of crystals. Figure 1 shows growth of $\mathrm{NdBP}$ in a crystallizer. The fully grown crystals were then removed from the crystallizing tube while washing in distilled water. The crystals were then dried and placed in the vacuum so that they do not come in contact with dust particles. In order to establish condition conducive for the growth of NdBP system, number of experiments were performed under varying conditions of different growth parameters viz., gel $\mathrm{pH}$, concentration of upper and lower reactant, gel ageing and gel molarity. The optimum conditions for the synthesis of NdBP system was: lower reactant concentration $\left(\mathrm{H}_{3} \mathrm{PO}_{4}\right): 0.5 \mathrm{M}$, upper reactant concentration (mixture of $\mathrm{BaCl}_{2}$ and $\mathrm{Nd}\left(\mathrm{NO}_{3}\right)_{3}$ ): $1 \mathrm{M}$, gel concentration $\left(\mathrm{Na}_{2} \mathrm{SiO}_{3}\right): 0.5 \mathrm{M}$, gel ageing: $96 \mathrm{~h}$, gel pH: 6 and growth temperature: $30-35^{\circ} \mathrm{C}$, whereas the optimum conditions for the synthesis of pure BP system as already reported $^{7}$ is same except that the upper reactant concentration $\left(\mathrm{BaCl}_{2}\right)$ was $0.5 \mathrm{M}$. 


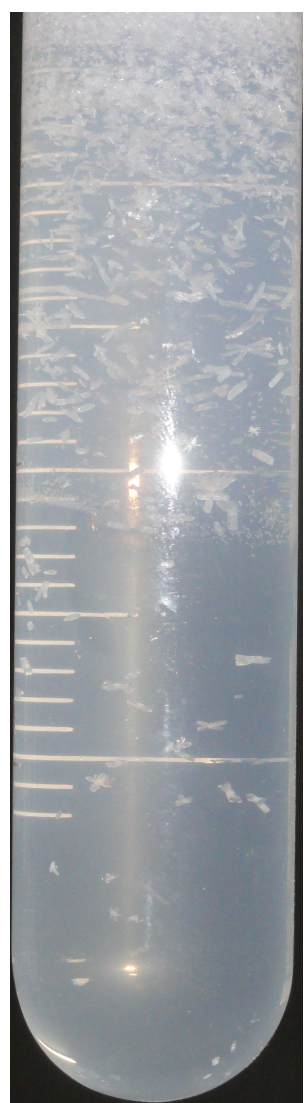

Fig. 1. Growth of NdBP system in a crystallizer.

It is interesting to find that $\mathrm{Nd}$ doping changes the morphology of the grown system. Figure 2 shows scanning electron micrograph of pure BP system exhibiting various types of morphology which includes platelets, cuboid, coalesced. However, Fig. 3 shows the flower-like growth of $\mathrm{NdBP}$ system, which clearly indicates the effect of $\mathrm{Nd}$ doping on the morphology of the grown system.

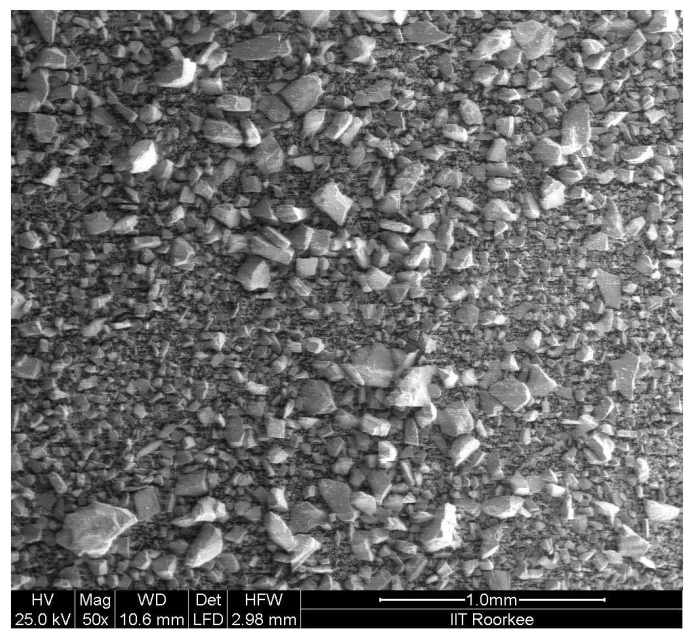

Fig. 2. Electron micrograph showing different morphologies exhibited by BP system.

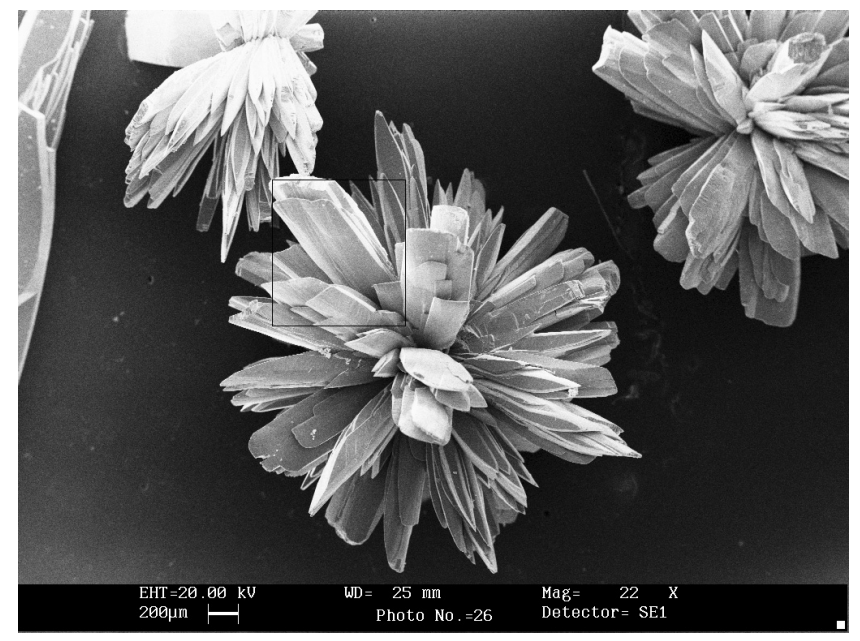

Fig. 3. Electron micrograph showing flower-like growth of NdBP system.

From the micrograph (Fig. 3), one can say that this flower type morphology is because of number of crystals aggregating to make it a spherullitic shape with each crystal having its own nuclei. It is interesting to see the features of these fibers at higher magnification, so highlighted portion of Fig. 3 shown in Fig. 4(a) reveals the growth of NdBP in the form of fibers. Figure 4(b) shows electron micrograph at still a higher magnification, where the tip of that fiber shows two different types of striations on the surface, one perpendicular to the growth layer and other parallel to the growth layer. Striations are basically growth-induced inhomogeneities in the crystal which are aligned along faceted or nonfaceted growth surface. In the present case, striations are defined as kinetic striations as they occur due to relatively high supersaturation.

\subsection{XRD analysis}

The powder XRD pattern of NdBP and BP system is shown in Figs. 5(a) and 5(b). The occurrence of sharp peaks at specific $2 \theta$ Bragg angles indicates the crystallinity of the grown system. There is a shift in the positions of peaks because of doping of $\mathrm{Nd}^{3+}$ ion in the composition of BP system. This indicates a change in the internal structure of the system due to the change in the bond length. Since the ionic radii of barium and neodymium are comparable, one can expect identical crystal structure for NdBP with that of BP system. The details of the XRD plot depicting interplanar $(d)$ spacing and corresponding [hkl] planes of NdBP and BP system is given in Table 1 . Single crystal XRD analysis establishes that NdBP belongs to orthorhombic crystal system as has been obtained in case of barium phosphate crystal. ${ }^{6}$ The lattice parameters obtained in case of NdBP are: $a=4.5930 \AA, b=14.1366 \AA, c=17.1682 \AA$ and $\alpha=\beta=$ $\gamma=90^{\circ}$ with space group Pmma (bca) and volume of unit 


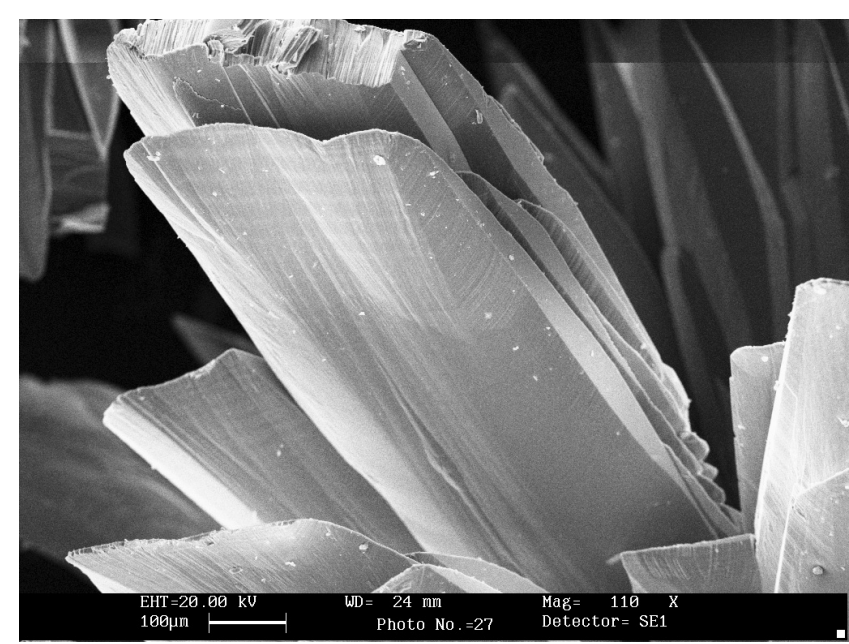

(a)

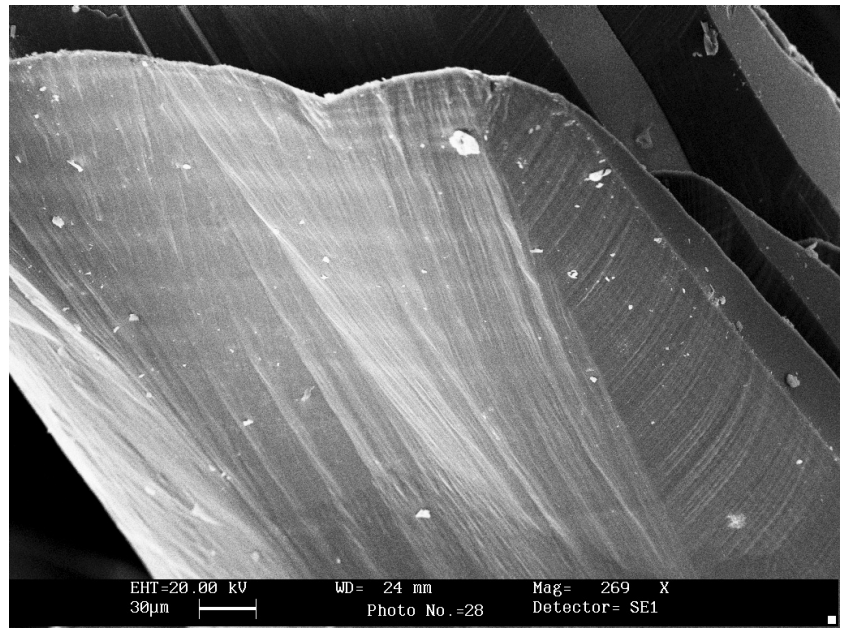

(b)

Fig. 4. (a) Electron micrograph at higher magnification showing highlighted portion of Fig. 3. (b) SEM micrograph of NdBP showing highlighted two different types of striations.

cell being $1114.72 \AA^{3}$. From the powder as well as single crystal diffraction data, it has been found that $\mathrm{NdBP}$ and $\mathrm{BP}$ system belong to orthorhombic crystal system.

\subsection{Energy dispersive $X$-ray analysis (EDAX)}

Qualitative and quantitative analysis for the elements present were performed by EDAX. The EDAX spectrum for NdBP and BP system is shown in Figs. 6(a) and 6(b). From the spectra, the peaks corresponding to all major elements are present along with neodymium thereby suggesting that $\mathrm{Nd}^{3+}$ ion has entered into the lattice of the BP system. The experimental and theoretical calculated atomic and weight percentage of elements in $\mathrm{NdBP}$ and BP system is given in Table 2. The values were calculated as per the formulas $\mathrm{Nd}_{3(0.1)} \mathrm{Ba}_{3(0.9)}\left(\mathrm{PO}_{4}\right)_{2} \cdot \mathrm{H}_{2} \mathrm{O}$ and $\mathrm{Ba}_{3}\left(\mathrm{PO}_{4}\right)_{2} \cdot \mathrm{H}_{2} \mathrm{O}$. The experimental and theoretical values are in good agreement with each other. The presence of water molecule was further confirmed by Fourier transform infrared analysis.

\subsection{Fourier transform infrared spectroscopy (FTIR)}

FTIR was widely used for the identification of chemical bonds, functional unit and internal structure of molecules present in a grown composition. ${ }^{9}$ The peaks were identified in comparison with standard data. A comparison of the bands and peaks of the FTIR spectra reveals interesting features. Figure 7 shows FTIR spectrum obtained in case of NdBP system and inset of Fig. 7 shows the spectrum for BP. The fundamental IR frequencies observed in other phosphate compounds has been obtained in the present case, which

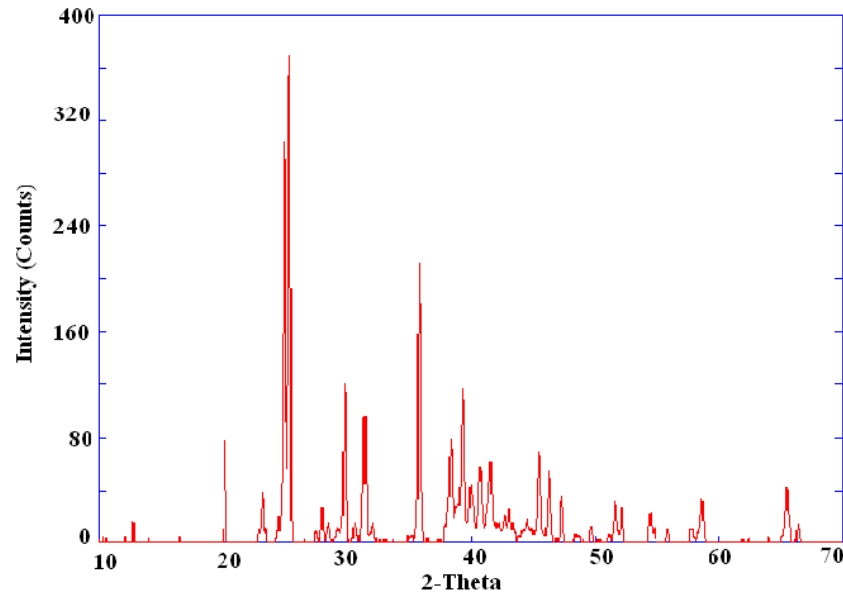

(a)

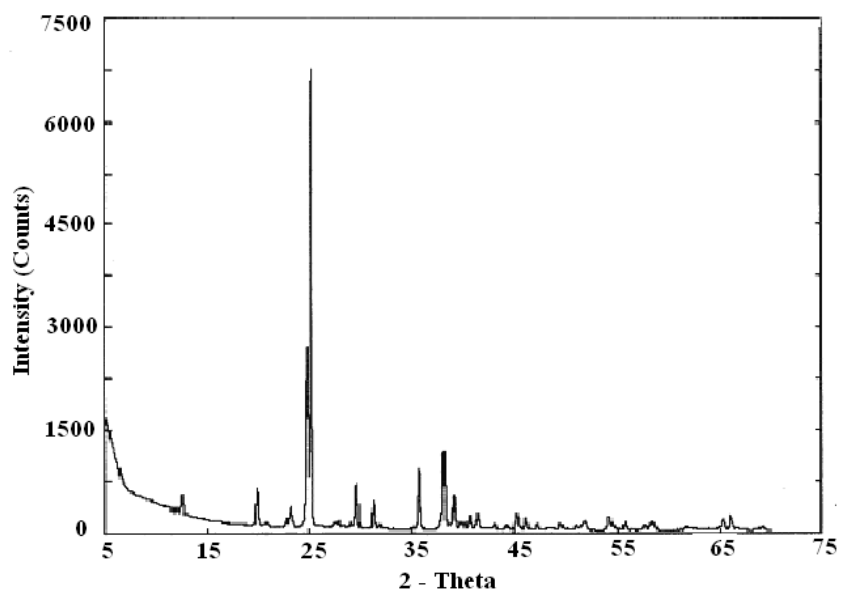

(b)

Fig. 5. Powder XRD pattern of (a) NdBP and (b) BP system. 
Table 1. Compiled data of various 'hkl' planes corresponding to different Bragg angles, interplanar spacing ' $d$ ' and intensity for NdBP and BP system.

\begin{tabular}{|c|c|c|c|c|c|c|c|}
\hline \multicolumn{2}{|c|}{$d$-Spacing $(\AA)$} & \multicolumn{2}{|c|}{$2 \theta$} & \multicolumn{2}{|c|}{ [hkl] planes } & \multicolumn{2}{|c|}{ Intensity (counts) } \\
\hline $\mathrm{NdBP}$ & $\mathrm{BP}$ & NdBP & $\mathrm{BP}$ & NdBP & $\mathrm{BP}$ & $\mathrm{NdBP}$ & BP \\
\hline 7.06 & 7.02 & 12.51 & 12.54 & 020 & 020 & 16 & 343 \\
\hline 4.43 & 4.44 & 20.08 & 19.96 & 101 & 101 & 82 & 633 \\
\hline 3.84 & 3.85 & 23.08 & 23.02 & 120 & 120 & 42 & 356 \\
\hline 3.57 & 3.58 & 24.85 & 24.82 & 103 & 103 & 38 & 2794 \\
\hline 3.51 & 3.52 & 25.34 & 25.26 & 122 & 122 & 374 & 6734 \\
\hline 3.005 & 3.00 & 29.7 & 29.69 & 043 & 043 & 120 & 725 \\
\hline 2.85 & 2.85 & 31.36 & 31.27 & 133 & 133 & 98 & 475 \\
\hline 2.46 & 2.52 & 35.67 & 35.59 & 045 & 143 & 217 & 1002 \\
\hline 2.34 & 2.36 & 38.26 & 38.08 & 144 & 054 & 83 & 1239 \\
\hline 2.29 & 2.29 & 39.22 & 39.21 & 200 & 200 & 124 & 580 \\
\hline
\end{tabular}

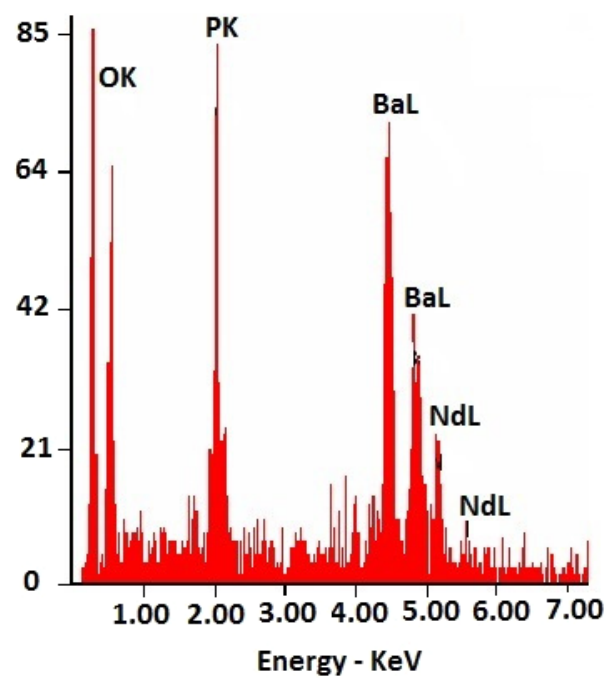

(a)

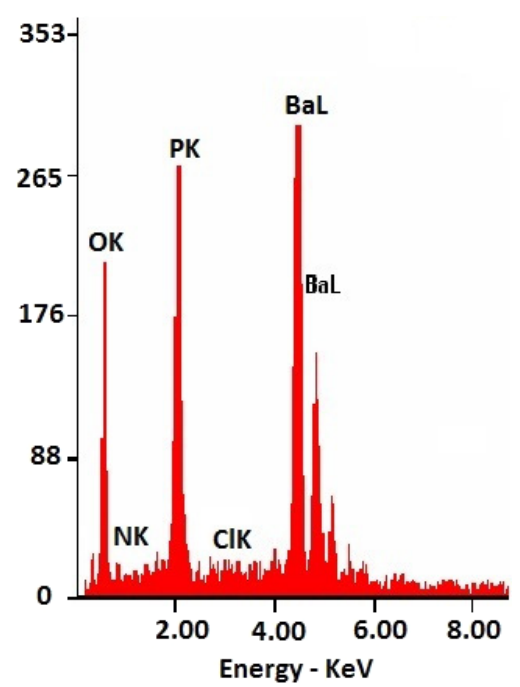

(b)

Fig. 6. EDAX pattern of (a) NdBP and (b) BP system.
Table 2. Experimental and theoretical calculated composition obtained from EDAX of various constituent elements present in case of $\mathrm{NdBP}$ and $\mathrm{BP}$ system.

\begin{tabular}{|c|c|c|c|c|c|c|c|c|}
\hline \multirow[b]{3}{*}{ Element } & \multicolumn{4}{|c|}{ Experimental values } & \multicolumn{4}{|c|}{ Theoretical values } \\
\hline & \multicolumn{2}{|c|}{ wt. $\%$} & \multicolumn{2}{|c|}{ at. $\%$} & \multicolumn{2}{|c|}{ wt. $\%$} & \multicolumn{2}{|c|}{ at. $\%$} \\
\hline & NdBP & BP & $\mathrm{NdBP}$ & BP & NdBP & BP & NdBP & BP \\
\hline Oxygen & 17.61 & 20.59 & 52.66 & 53.83 & 23.2 & 22.65 & 64.28 & 59.5 \\
\hline Phosphorus & 15.67 & 14.75 & 24.21 & 19.91 & 9.9 & 9.74 & 14.28 & 13.22 \\
\hline Barium & 60.43 & 61.91 & 21.05 & 18.85 & 59.8 & 64.85 & 19.28 & 19.84 \\
\hline Neodymium & 6.29 & - & 2.09 & - & 6.97 & - & 2.14 & - \\
\hline
\end{tabular}

confirms the presence of phosphate group in the grown system. The presence of band at 3486.7 and $3431.5 \mathrm{~cm}^{-1}$ for $\mathrm{NdBP}$ and BP respectively indicates the presence of water of hydration in the system. This supports the inference from the EDAX analysis that the grown system has one water molecule. The peaks corresponding to other functional groups are also present in the grown composition.

The peak observed at 2366.9 and $2381.9 \mathrm{~cm}^{-1}$ is attributed to the presence of $\mathrm{Si}-\mathrm{H}$ bond in the $\mathrm{NdBP}$ and $\mathrm{BP}$ systems respectively. Another peak observed at 1073.8 and $1074.9 \mathrm{~cm}^{-1}$ in BP and NdBP may be due to the fundamental stretching vibration frequency of the phosphate ions. Moreover, the peaks observed at 531.5, 426.0, 530.4 and $429.2 \mathrm{~cm}^{-1}$ are due to metal oxygen bonds. Fujitha et al. ${ }^{10}$ have suggested that the metal oxygen bands will appear around $800 \mathrm{~cm}^{-1}$. Morris, ${ }^{11}$ Gibson and Stump ${ }^{12}$ have proposed the presence of another metal oxygen bands at $500 \mathrm{~cm}^{-1}$. Comparison of the FTIR spectrum of NdBP with that of pure BP reveals a slight shift in some of the characteristically vibrational frequency because of $\mathrm{Nd}$ doping. It could be due to the lattice strain developed because of doping. The shift in the wave number was due to the difference in mass number of neodymium (144.24) with that of barium (137.34) ions. As reported in the literature, ${ }^{13}$ the difference in mass of ions leads to a change in the molecular geometry and mechanical vibrations, which results in shift of bands. A comparative assignment of prominent peaks of FTIR spectra is given in Table 3.

\subsection{Electrical properties}

Electrical properties which include dielectric studies on $\mathrm{NdBP}$ have been carried out to study the response of electric field and effect of doping on the dielectric behavior of the system. The study of dielectric characteristics indicates the response of the grown material to an applied electric field. Variation of dielectric constant $\left(\varepsilon^{\prime}\right)$ can be attributed to different types of polarization, which comes into play at different stages of its response to varying temperature and frequency of the applied alternating field. The dependence of 


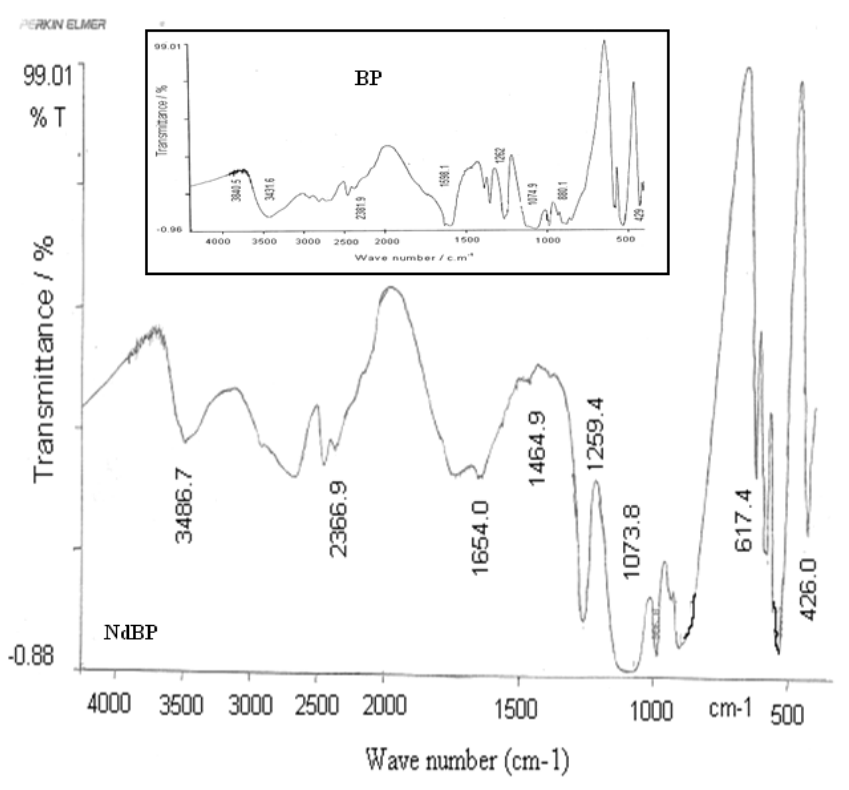

Fig. 7. FTIR spectrum of the NdBP system crystal depicting the various functional groups present along with FTIR spectra for BP shown in inset.

dielectric constant $\left(\varepsilon^{\prime}\right)$ and loss tangent $(\tan \delta)$ on frequency of the applied ac field $(5 \mathrm{kHz}-1 \mathrm{MHz})$ was studied in the temperature range of $40-420^{\circ} \mathrm{C}$.

\subsubsection{Dependence of dielectric constant and tangent loss on temperature}

Figure 8(a) shows the variation of dielectric constant $\left(\varepsilon^{\prime}\right)$ with temperature as a function of frequencies $(5 \mathrm{kHz}-1 \mathrm{MHz})$ for NdBP. The dielectric curve shows two anomalies, one at $67^{\circ} \mathrm{C}$ and other at $387^{\circ} \mathrm{C}$. The value of $\varepsilon^{\prime}$ increases with increase in temperature, attaining maximum value first at $67^{\circ} \mathrm{C}$ and then at $387^{\circ} \mathrm{C}$. Such type of behavior was observed for other materials which exhibit two transitions. ${ }^{14,15}$ The first $\left(67^{\circ} \mathrm{C}\right)$ being attributed to the absorption of water $\left(\mathrm{H}_{2} \mathrm{O}\right)$ molecule whereas the second $\left(387^{\circ} \mathrm{C}\right)$ is the phase transition of the NdBP system. The maximum value of $\varepsilon^{\prime}$ obtained at $5 \mathrm{kHz}$ was 25 for first transition $\left(67^{\circ} \mathrm{C}\right)$, whereas for

Table 3. Presence of various functional groups in case of NdBP and BP system.

\begin{tabular}{lcc}
\hline Assignments of bands/peaks & Peaks for NdBP & Peaks for BP \\
\hline O-H symmetric and asymmetric & 3486.7 & 3431.5 \\
$\quad$ stretching & 2366.9 & 2381.9 \\
Si-H bond & 1469.9 & 1598.1 \\
H-O-H bending & 1259.4 & 1262 \\
P = O stretching & $986.8,899.2$ & $987.3,880.1$ \\
P-O stretching & 578.5 & 578.4 \\
P-P Stretching & $531.5,426.0$ & $530.4,429.2,405.5$ \\
Metal-Oxy bond & & \\
\hline
\end{tabular}

second transition $\left(387^{\circ} \mathrm{C}\right)$ the maximum value was 47 . In this case, no drift in transition temperature was observed. The temperature variation of $\varepsilon^{\prime}$ values in case of NdBP was compared with pure BP system as shown in Fig. 8(b). Comparing these results, the following interesting observations were obtained.

The value of $\varepsilon^{\prime}$ is higher in case of BP as compared to $\mathrm{NdBP}$. For example, for $5 \mathrm{kHz}$ the value of $\varepsilon_{\max }^{\prime}$. in case of $\mathrm{BP}$ is 64 whereas in case of $\mathrm{NdBP}$ it is 47 , thereby suggesting that dielectric constant decreases with the increase in substitution. In Nd doping, there are two suggested transitions instead of single phase transition for BP. For NdBP, the ferroelectric-paraelectric phase transition occurs at $387^{\circ} \mathrm{C}$ whereas for BP the phase transition is in the range of 368 $383^{\circ} \mathrm{C}$. The dielectric studies on pure BP system suggests that the material exhibit relaxor behavior in its pure form but when it is doped with neodymium then the relaxor behavior is completely lost and material act as simple dielectric material. This means that Nd doping suppresses the relaxor characteristics of the material.

The variation of dielectric loss $(\tan \delta)$ with temperature for NdBP system exhibits similar behavior to that of variation of dielectric constant $\left(\varepsilon^{\prime}\right)$ with temperature. Figure 9 shows variation of dielectric loss $(\tan \delta)$ with temperature where loss is observed in the temperature range of $290-420^{\circ} \mathrm{C}$. The peak value of dielectric loss at temperature of $376^{\circ} \mathrm{C}$ is $4.5,2.7$, 1.76 and 0.74 for frequencies of $5,10,20$ and $100 \mathrm{kHz}$, respectively.

\subsubsection{Diffusivity study}

The region around the dielectric peak is broadened so we can say that there is a diffuse phase transition. The diffuse phase transition in ferroelectric is characterized by extending the phase transition in a wide temperature interval around the transition temperature where the dielectric constant assumes its maximum value. The diffuse phase transition does not follow Curie Weiss law but exhibits the temperature dependence. The degree of diffusivity $(\gamma)$ can be calculated using the expression

$$
1 / \varepsilon^{\prime}-1 / \varepsilon_{\max }^{\prime}=A\left(T-T_{c}\right)^{\gamma} .
$$

Here $\varepsilon_{\max }^{\prime}$ is the peak value of the dielectric constant and $\gamma$ is the critical exponent which lies in the range $1<\gamma \leq 2$. For ideal Curie-Weiss behavior $\gamma=1$, whereas if $\gamma$ is between 1 and 2 , it indicates diffused behavior.

Figure 10 shows the variation of $\ln \left(1 / \varepsilon^{\prime}-1 / \varepsilon_{\max }^{\prime}\right)$ with $\ln \left(T-T_{c}\right)$ for frequency of 5 and $10 \mathrm{kHz}$ for NdBP. The value of ' $\gamma$ ' was estimated from the slope of the curves. The value of ' $\gamma$ ' at a frequency of 5 and $10 \mathrm{kHz}$ was found to be equal to 1.49 and 1.35 whereas for BP system the value of ' $\gamma$ ' comes out to be 1.5 for $5 \mathrm{kHz}$ and 1.34 for $10 \mathrm{kHz}$. $^{7}$ Thus, in both pure as well as in Nd-doped system the value of diffusing coefficient ' $\gamma$ ' is of almost the same order. This diffuseness may be due to the compositional fluctuations and 


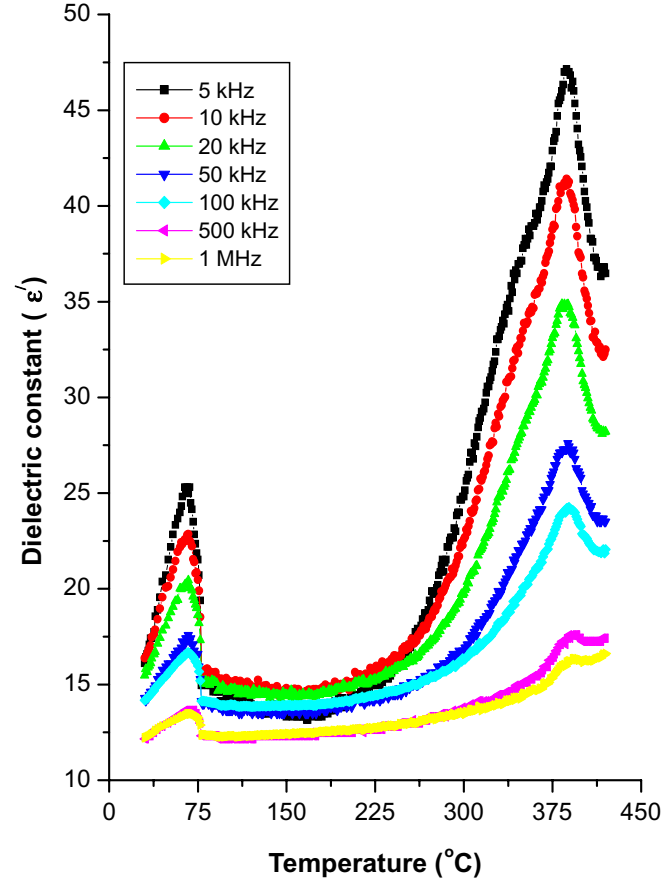

(a)

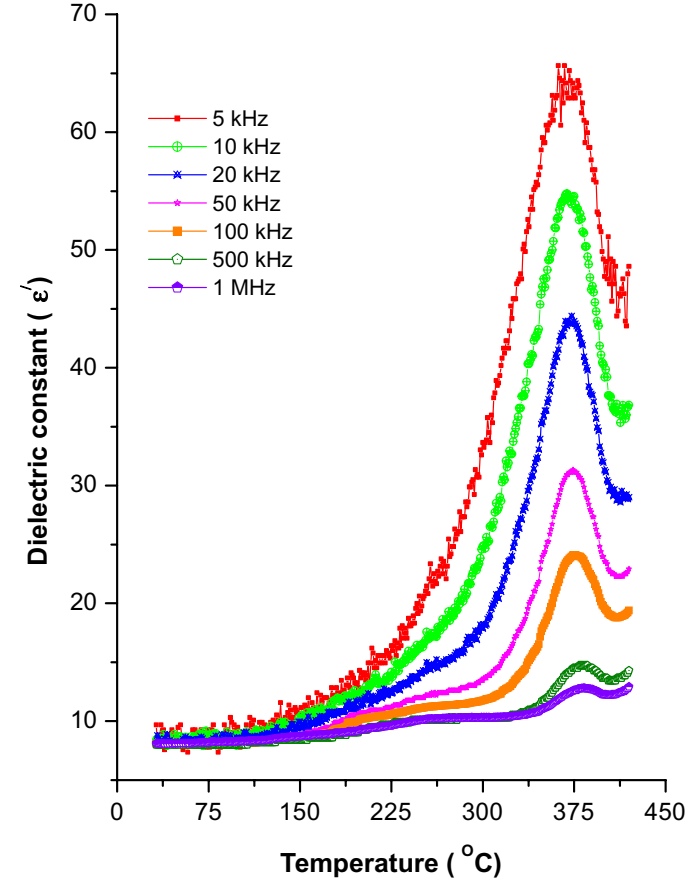

(b)

Fig. 8. (a) Plot showing variation of dielectric constant with temperature for different frequencies in case of NdBP system. (b) Graph showing variation of dielectric constant with temperature for different frequencies in case of BP system.

structural disordering in the arrangement of cations in one or more crystallographic sites of the structure. ${ }^{16,17}$ This suggests a microscopic inhomogeneity in the material under study

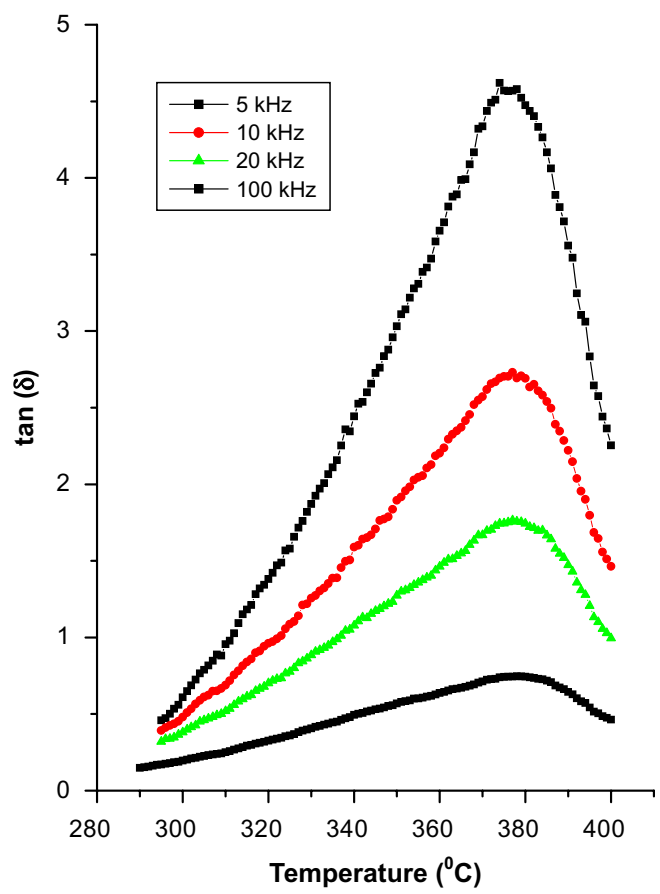

Fig. 9. Plot showing variation of dielectric loss with temperature for different frequencies in NdBP. with local curie points. Such type of behavior has been reported for many other materials. ${ }^{18-20}$

\subsubsection{AC conductivity and activation energy}

The ac conductivity was measured at different frequencies of $5,10,20$ and $100 \mathrm{kHz}$ in the temperature range of $300-420^{\circ} \mathrm{C}$ for NdBP systems. Figure 11(a) shows variation of ac conductivity ' $\ln \left(\sigma_{\mathrm{ac}}\right)$ ' with temperature. The graph indicates

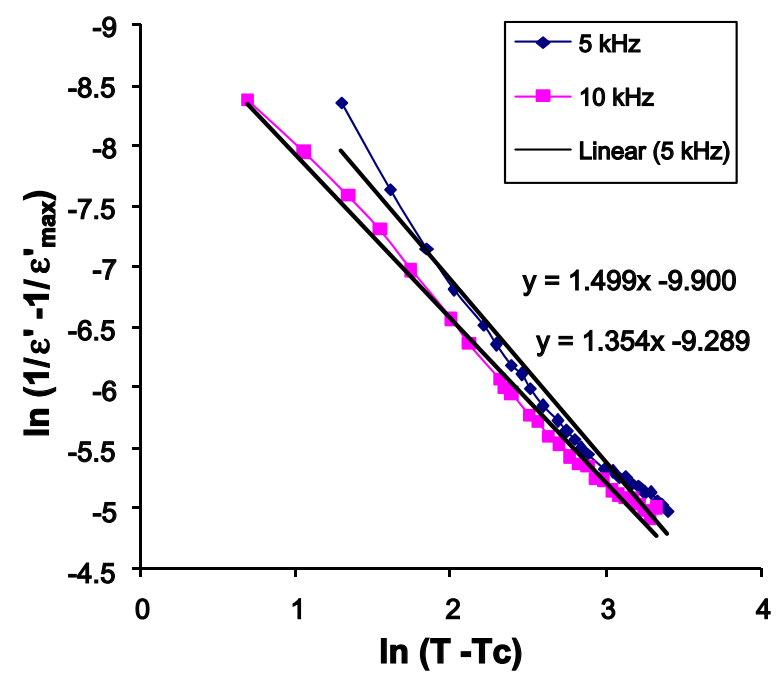

Fig. 10. $\ln \left(1 / \varepsilon^{\prime}-1 / \varepsilon_{\max }^{\prime}\right)$ versus $\ln \left(T-T_{c}\right)$ curves at 5 and $10 \mathrm{kHz}$ for diffuse phase transition in case of NdBP. 


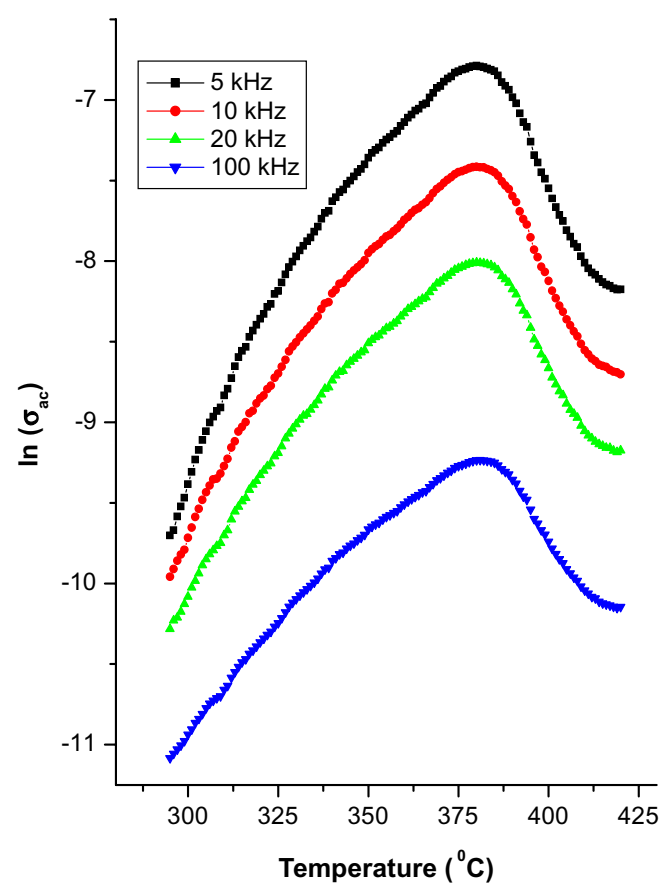

(a)

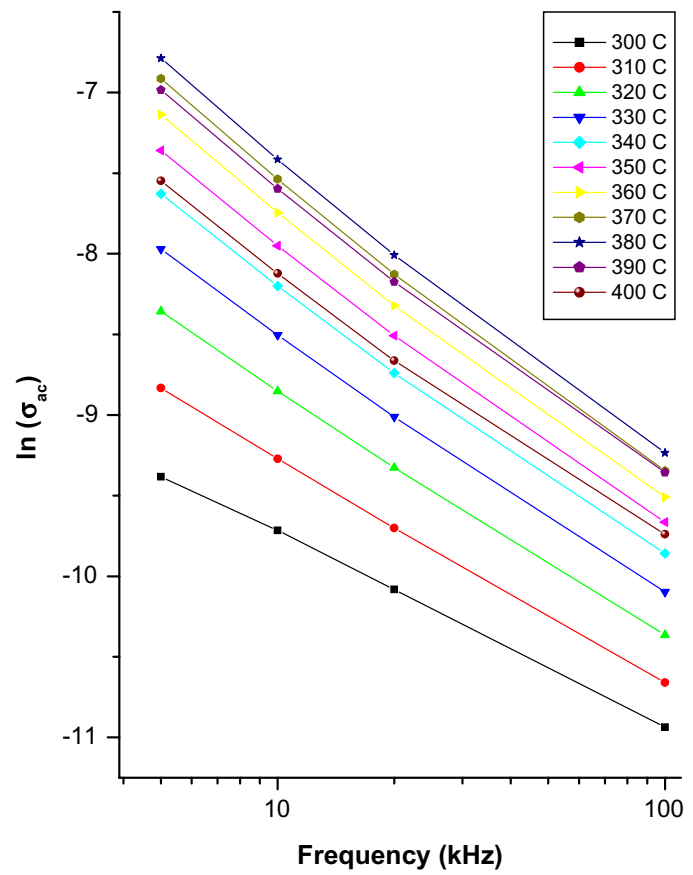

(b)

Fig. 11. (a) Graph showing variation of $\ln \sigma_{\mathrm{ac}}$ with frequency at different temperatures for NdBP. (b) Variation of $\ln \sigma_{\mathrm{ac}}$ with frequency at different temperatures for $\mathrm{NdBP}$.

strong dependence of ' $\ln \left(\sigma_{\mathrm{ac}}\right)$ ' on both temperature as well as frequency of the applied field as has been reported for other materials also. ${ }^{21,22}$ It was observed from the plot of ' $\ln \left(\sigma_{\mathrm{ac}}\right)$ ' versus temperature that conductivity increases with the increase of temperature and reaches a maximum value and then further decreases with the rise in temperature. The graph shows that maximum value of conductivity was obtained at Curie temperature. The influence of conductivity has been explained by considering the mobility of charge carrier responsible for hopping. As temperature increases, the mobility of hopping ions also increases thereby increasing conductivity. The variation of ac conductivity ' $\ln \left(\sigma_{\mathrm{ac}}\right)^{\prime}$ with frequency at various temperatures ranging from $300^{\circ} \mathrm{C}$ to $420^{\circ} \mathrm{C}$ is shown in Fig. 11(b). From the graph, it was observed that ac conductivity increases with increase in frequency at each temperature thus confirming polaron hopping. ${ }^{23,24}$ This frequency dependence of $\ln \left(\sigma_{\mathrm{ac}}\right)$ has been explained with the help of Maxwell Wagner model. ${ }^{25,26}$ According to this model, at lower frequencies, the hopping frequency of electrons between $\mathrm{Ba}^{2+}$ and $\mathrm{Nd}^{3+}$ ions is less at the metal/insulator interface surface. With the increase in the frequency of the applied field, the conduction mechanism become more active by promoting the hopping of charge carriers between $\mathrm{Ba}^{2+}$ and $\mathrm{Nd}^{3+}$ ions thereby increasing the hoping frequency. This observed frequency dependence of relative permittivity can also be explained using Jonsher's universal dielectric response (UDR) model ${ }^{27,28}$ in case of heterogeneous materials. The frequency dispersion of conductivity was observed throughout the region in all the temperature range thereby suggesting combination of resistor and capacitor network with power law dependence of conductivity thereby characterizing hopping conduction mechanism. The value of ' $s$ ' as obtained from power law is given in Table 4.

$$
\sigma_{\mathrm{ac}}(\omega) \sim(\omega)^{s} \text { with } 0 \leq s \leq 1 .
$$

The variation of $\ln \sigma_{\mathrm{ac}}$ with $1000 \mathrm{~T}^{-1}$ is shown in Fig. 12 and from the curve one observes that there is a change in the slope at a temperature which is almost same as that of the transition temperature. This change in slope is due to the difference in activation energy in the paraelectric and ferroelectric phase. There are distinct region of conduction mechanisms in different temperature ranges. (i) n-and/or p-type hopping charge (corresponding to low temperature), (ii) small polarons and oxygen vacancy conduction (corresponding to

Table 4. Value of ' $s$ ' at different temperatures for NdBP system.

\begin{tabular}{lcc}
\hline S. No. & Temperature $\left({ }^{\circ} \mathrm{C}\right)$ & Value of ' $s$ \\
\hline 1 & 300 & 0.47 \\
2 & 320 & 0.33 \\
3 & 340 & 0.25 \\
4 & 360 & 0.21 \\
5 & 380 & 0.18 \\
6 & 400 & 0.27 \\
7 & 420 & 0.34 \\
\hline
\end{tabular}




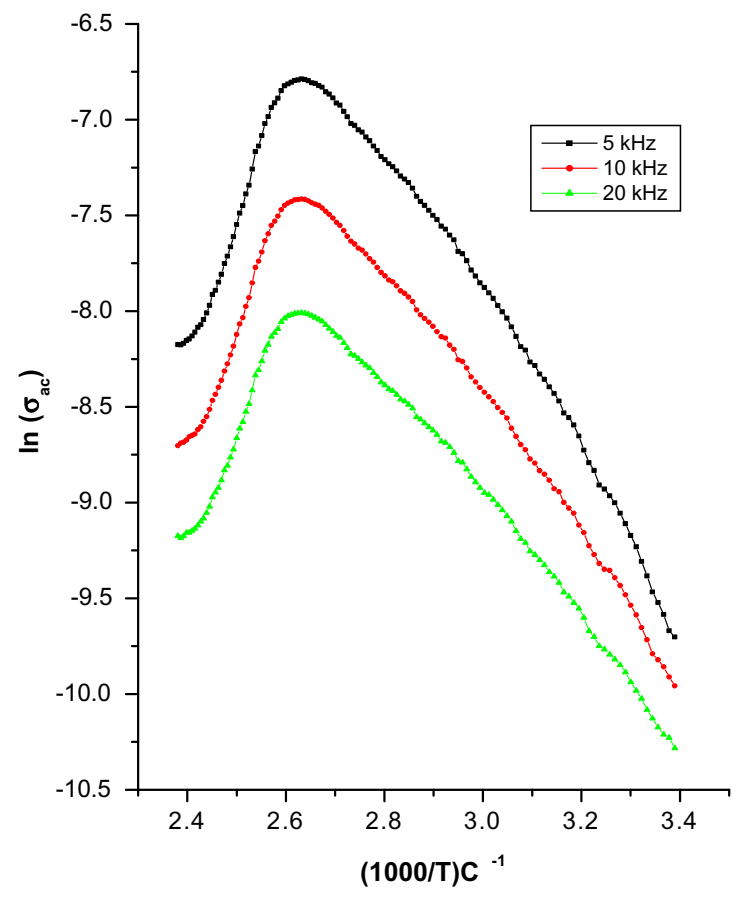

Fig. 12. Plot showing variation of $\ln \sigma_{\mathrm{ac}}$ with $1000 \mathrm{~T}^{-1}$ at three different frequencies viz., 5, 10 and $20 \mathrm{kHz}$ for NdBP.

intermediate temperature region) and (iii) intrinsic ionic conduction (at high temperature region). ${ }^{29}$ The nature of variation of ac conductivity $\left(\sigma_{\mathrm{ac}}\right)$ for wide temperature range favors the thermally activated transport properties obeying Arrhenius formula. The activation energy for NdBP is calculated from Arrhenius plot of ' $\ln \sigma_{\mathrm{ac}}$ ' using the relation

$$
\sigma_{\mathrm{ac}}=\sigma_{0} \exp \left(-E_{a} / K_{B} T\right),
$$

where ' $\sigma_{\mathrm{ac}}$ ' is the conductivity at temperature ' $T$ ', ' $E_{a}$ ' is the activation energy for the electrical process and ' $K_{\mathrm{B}}$ ' is the Boltzman constant.

Activation energy was calculated from the slope of the straight line in the ferroelectric region of Fig. 12 showing the variation of $\ln \sigma_{\mathrm{ac}}$ with $1000 T^{-1}$. The activation energy for frequencies of 5,10 and $20 \mathrm{kHz}$ was calculated and is given in Table 5 for both NdBP and BP system. From the values of activation energies, it is clear that as the frequency increases, the value of activation energy decreases. This type of behavior suggests that the conduction mechanism may be due to the hopping of the charge carriers (i.e., $\mathrm{n}$ or $\mathrm{p}$ type) from one

Table 5. Activation energy calculated for three different frequencies of 5,10 and $20 \mathrm{kHz}$ for NdBP and BP system.

\begin{tabular}{lccc}
\hline S. No. & Frequency $(\mathrm{kHz})$ & NdBP & BP \\
\hline 1 & 5 & 0.32 & 0.21 \\
2 & 10 & 0.29 & 0.18 \\
3 & 20 & 0.26 & 0.16 \\
\hline
\end{tabular}

site to another. Therefore, a very small amount of energy is required to activate the charge carriers/electrons for electrical conduction. It has been shown by Ang et al. ${ }^{22}$ and Moretti and Calendini ${ }^{30}$ that the value of activation energy depends on ionization level of the oxygen vacancy. Usually activation energy less than $1 \mathrm{eV}$ is connected to singly ionized vacancies $^{22}$ and or electronic mobility in space charge region. ${ }^{31}$ Thus, the conduction process within this temperature range may be due to the hopping of charge carriers and/or singly oxygen vacancies.

\section{Conclusion}

Based on the results, the following conclusions can be drawn:

- NdBP crystals were synthesized in the form single crystal by room temperature solution technique, i.e., gel encapsulation technique. The crystal NdBP belongs to orthorhombic system with lattice parameter: $a=4.5930 \AA$, $b=14.1366 \AA, c=17.182 \AA$ and $\alpha=\beta=\gamma=90^{\circ}$.

- Microstructural investigations reveal that pure BP grows as single crystal with different morphologies which includes platelets, cuboids, coalesced whereas $\mathrm{Nd}$ doping completely changes the morphology of the grown system. NdBP crystals grow as aggregates forming a flower-like shape with each crystal having its own nuclei. At higher magnification, tip of the fiber shows two different types of striations, one perpendicular to the growth layer and other parallel to the growth layer which is due to relatively high supersaturation. The stoichiometric compositions of the grown system as established by EDAX technique is $\mathrm{Nd}_{3(0.1)} \mathrm{Ba}_{3(0.9)}\left(\mathrm{PO}_{4}\right)_{2} \cdot \mathrm{H}_{2} \mathrm{O}$. FTIR results support the presence of water of hydration and other functional groups in NdBP system.

- Nd doping in barium phosphate decreases the maximum value of dielectric constant $\left(\varepsilon^{\prime}\right)$ from 64 to 47 at a frequency of $5 \mathrm{kHz}$. NdBP shows two transitions in comparison to single transition for BP. The value of transition temperature $\left(T_{c}\right)$ in case of $\mathrm{NdBP}$ is $387^{\circ} \mathrm{C}$ which remains same for all the frequencies considered whereas in case of $\mathrm{BP}$ the value of $T_{c}$ is in the range of $367-383^{\circ} \mathrm{C}$ for different frequencies considered. This suggests relaxor behavior for BP system whereas $\mathrm{Nd}$ doping suppresses the relaxor characteristics of the material.

- The value of diffusing coefficient $(\gamma)$ for 5 and $10 \mathrm{kHz}$ is 1.49 and 1.35 for NdBP whereas for BP the values are 1.5 and 1.34, respectively. This suggests a microscopic inhomogeneity in the material under study with different local Curie points. The increase in conductivity with frequency obeys UDR model showing dependence of power law. The value of activation energy in case of $\mathrm{NdBP}$ at frequencies of 5,10 and $20 \mathrm{kHz}$ are $0.32,0.29$ and $0.26 \mathrm{eV}$, respectively. The value of activation energy decreases with increase in frequency, suggesting conduction mechanism is due to hopping of the charge carriers from one site to another. 


\section{References}

${ }^{1}$ H. Koelmans and A. P. M. Cox, Luminescence of modified tinactivated strontium orthophosphate, J. Electro Chem. Soc. 104, 442 (1957).

${ }^{2}$ N. Zaitseva and L. Carman, Rapid growth of KDP type crystals, Prog. Cryst. Growth Character. Mater. 41, 1 (2001).

${ }^{3}$ S. A. Devries, P. Goedtkindt, W. J. Huisman, M. J. Zwanenburg, R. Feidenhans, S. L. Bennett, D. M. Smilgus, A. Stierle, J. J. De Yoreoo, W. J. P. Van Enckevort, P. Bennema and E. Vlieg, X-ray diffraction studies of potassium dihydrogen phosphate (KDP) crystal surfaces, J. Cryst. Growth 205, 202 (1999).

${ }^{4}$ E. Banks, R. Chianelli and F. Pintchovsky, The growth of some alkaline earth orthophosphates in gelatin gel, J. Cryst. Growth 18, 185 (1973).

${ }^{5}$ T. F. Murray and R. H. Dungan, Oxygen firing can replace hot pressing for PZT, Ceram. Ind. 82, 74 (1964).

${ }^{6}$ S. Suri, K. K. Bamzai and V. Singh, Growth and thermal kinetics of pure and cadmium doped barium phosphate single crystal, J. Therm. Anal. Calorimetry 105, 229 (2011).

${ }^{7}$ S. Suri, K. K. Bamzai and V. Singh, Dielectric and conductivity studies on pure and cadmium doped barium phosphate crystals, Ferroelectrics 423, 94 (2011).

${ }^{8}$ E. J. W. Verwey and E. L. Heilman, Physical properties and cation arrangement of oxides with spinel structures I. Cationic arrangement in solids, J. Chem. Phys. 15, 174 (1947).

${ }^{9}$ N. B. Colthup, L. H. Daly and S. E. Wiberley, Introduction to Infrared and Raman Spectroscopy, 3rd edn. (Academic Press, 1990).

${ }^{10}$ J. Fujitha, A. E. Martell and K. Nakamoto, Infrared spectra of metal chelete compounds IV. A normal coordinate treatment of oxalate metal compound, J. Chem. Phys. 36, 324 (1962).

${ }^{11}$ D. E. Morris and D. E. Hobart, Raman spectra of lanthanide oxalates, J. Raman Spectrosc. 19, 231 (1988).

${ }^{12}$ J. K. Gibson and N. A. Stump, Spectroscopic investigation of the thermal decomposition of europium oxalate, Thermochim. Acta 226, 301 (1993).

${ }^{13}$ S. J. Joshi, B. B. Parekh, K. D. Vohra and M. J. Joshi, Growth and characterization of gel grown pure and mixed iron - manganese levo tartrate crystals, Bull Mater. Sci. 29, 307 (2006).

${ }^{14} \mathrm{~F}$. Sandy and R. V. Jones, Dielectric relaxation of Rochelle salt, Phys. Rev. 168, 481 (1968).

${ }^{15}$ S. Kamba, J. Kulda, V. Petricek, G. Mclntyre and J. P. Kiat, High pressure structural and dielectric studies of the phase transitions in lithium thallium tartrate monohydrate, J. Phys. Condens. Mater. 14, 4045 (2002).
${ }^{16}$ R. N. P. Choudhary, S. R. Shannigrahi and A. K. Singh, Ferroelectric phase transition in $\mathrm{Ba}_{5} \mathrm{RTi}_{3} \mathrm{Nb}_{7} \mathrm{O}_{30}(\mathrm{R}=\mathrm{Nd}, \mathrm{Eu}, \mathrm{Gd})$, Bull Mater. Sci. 22, 975 (1999).

${ }^{17}$ H. R. Rukmini, R. N. P. Choudhary and D. L. Prabhakara, Sintering temperature dependent ferroelectric phase transistion of $\mathrm{Pb}_{0.91}\left(\mathrm{La}_{1-z / 3} \mathrm{Li}_{z}\right)_{0.09}\left(\mathrm{Zr}_{0.65} \mathrm{Ti}_{0.35}\right)_{0.9775} \mathrm{O}_{3}$, J. Phys. Chem. Solids 61, 1735 (2000).

${ }^{18}$ H. R. Rukmini, R. N. P. Choudhary and V. V. Rao, Diffuse phase transistion in $\mathrm{Pb}_{0.91}\left(\mathrm{La}_{1-z / 3} \mathrm{Li}_{z}\right)_{0.09}\left(\mathrm{Zr}_{0.65} \mathrm{Ti}_{0.35}\right)_{0.9775} \mathrm{O}_{3}$ ceramics, J. Phys. Chem. Solids 59, 1541 (1998).

${ }^{19}$ S. Sharma, R. Sati, R. N. P. Choudhary and T. P. Sinha, Diffuse phase transition in solid solution of $\mathrm{Pb}\left(\mathrm{Mg}_{1 / 4} \mathrm{Zn}_{1 / 4} \mathrm{Nb}_{1 / 2}\right) \mathrm{O}_{3-\delta}$ and $\mathrm{PbTiO}_{3}$, Mater. Lett. 16, 281 (1993).

${ }^{20} \mathrm{~K}$. Prasad, Diffuse phase transition in perovskite ferroelectrics, Indian J. Eng. Matter Sci. 7, 446 (2000).

${ }^{21} \mathrm{~S}$. Bera and R. N. P. Choudhary, SEM and electrical properties of $\mathrm{Pb}\left(\mathrm{Li}_{1 / 4} \mathrm{Y}_{1 / 4 W} \mathrm{~N}_{1 / 2}\right) \mathrm{O}_{3}$, Mater. Lett. 22, 197 (1995).

${ }^{22} \mathrm{C}$. Ang, $\mathrm{Z}$. Yu and L. E. Cross, Oxygen-vacancy related low frequency dielectric relaxation and electrical conduction in $\mathrm{Bi}$ : $\mathrm{SrTiO}_{3}$, Phys. Rev. B 62, 228 (2000).

${ }^{23} \mathrm{D}$. Alder, Insulating and metallic states in transition metal oxides, Solid State Phys. 21, 1 (1968).

${ }^{24}$ I. G. Austin and N. F. Mott, Polarons in crystalline and noncrystalline materials, Adv. Phys. 18, 41 (1969).

${ }^{25} \mathrm{P}$. K. Larsen and R. Metselaar, Electrical and dielectical properties of polycrystalline Yttrium Iron garnet: Space charge limited currents in an homogeneous solid, Phys. Rev. 8, 2016 (1973).

${ }^{26}$ A. K. Jonscher, Dielectric Relaxation in Solids (Chelsa Dielectrics, London, 1983).

${ }^{27}$ A. K. Jonscher, Physical basis of dielectric loss, Nature 253, 717 (1975).

${ }^{28}$ C. R. Bowen and D. P. Almond, Modelling the universal dielectric response in heterogeneous material using microstructural electrical networks, Mater. Sci. Technol. 22, 719 (2006).

${ }^{29}$ O. Raymond, R. Font, N. Suarez-Almodovar and J. Portelles, Frequency temperature response of the ferroelectric magnetic $\mathrm{Pb}$ $(\mathrm{Fe} 1 / 2 \mathrm{Nb} 1 / 2) \mathrm{O}_{3}$ ceramics obtained by different precursors, J. Appl. Phys. 97, 084108 (2005).

${ }^{30} \mathrm{P}$. Moretti and M. Calendini, Impurity energy levels and stability of $\mathrm{Cr}$ and $\mathrm{Mn}$ ions in Cubic $\mathrm{BaTiO}_{3}$, Phys. Rev. B 36, 3522 (1987).

${ }^{31}$ M. A. Omer, Elementary Solid State Physics: Principles and Applications (Addison-Wesley, Reading, 1975). 